\title{
Spatial reversal learning in rats and gerbils*
}

\author{
MAUREEN A. CAREY and GLORIA J. FISCHER \\ U'ashington State Liniversity. Pullman, Wash. 99163
}

Adult hooded rats and gerbils were trained to criterion on a spatial discrimination in a T-maze, followed by 15 daily 10 -trial reversals. Results indicated that both species improved significantly over the 15 reversals, but there was a reliable difference between species. Speqifically, gerbils made slightly more errors than did rats. Gerbils also were observed to explore in the maze more than rats. Present and prior findings suggest that gerbils perform less well than domestic rats on appetitive learning tasks. Other findings suggest that they are as good or better than rats at active avoidance learning. One possibility is that the gerbil's greater tendency to explore and/or their higher activity level interferes with performance on appetitive tasks but enhances performance in active avoidance learning.

Interest in the gerbil as an experimental animal has stimulated research on their learning ability compared with that of other rodent species. When compared on active avoidance tasks, gerbils have been found to be as good or better than rats (Ashe \& McCain, 1972; Boice, Boice, \& Dunham, 1968; Powell, 1972). However, on passive avoidance learning (Lippman, Galosy, \& Thompson, 1970) and on appetitive learning tasks (Carey \& Fischer, 1971; Wise \& Parker, 1968), gerbils were found to be inferior to rats. The present paper is a fuller account of the Carey \& Fischer (1971) study, in which gerbils were found to be slightly inferior to hooded rats on successive spatial reversal learning. It also advances the view that gerbil performance, relative to that of rats, may be a function of how the gerbil's greater tendency to explore and/or activity level (e.g., Lippman et al, 1970; Thompson \& Lippman, 1972) affects (i.e., interferes with or enhances) acquisition on a given task.

\section{METHOD}

\section{Subjects}

The Ss were three male and three female Long-Evans hooded rats (Rattus domesticus) and three male and three female Mongolian gerbils (Meriones unguiculatus). All were purchased from the same commercial source. At the beginning of training. the males were 90-100 day's of age and the females were 110-120 days of age. The animals were caged in threes. by sex and by species.

\section{Apparatus}

A transparent plastic $T$-maze had three $11 \times 3 \frac{11}{4} \times 5$ in. sections. separated by transparent guillotine doors. An opaque foodcup at the end of each alley was hidden from the animal's view by a 1-in. strip of masking tape stretched across the alley.

Procedure

The 12 Ss were run in two successive sets of six Ss (males.

*This experiment was a Senior Honors Thesis under the direction of the junior author. Results were presented in abstract form at the meeting of the Psychonomic Society. November, 1971 , in St. Louis. then females). For 10 days prior to training. they were adapted to a 22-h schedule of food deprivation. Pretraining consisted of a 20-min period of adaptation to the maze (Day 1), then two turning preference trials on Day 2. Spatial discrimination training was begun on Day 3. This consisted of 10 daily noncorrection trials, with food available on the S's nonpreferred side. On a given trial, all doors were closed behind the $S$, and a correct response resulted in three $45-\mathrm{mg}$ Noves pellets for rats and one $45-\mathrm{mg}$ pellet for gerbils. (The 3:1 ratio of food reward represented comparable proportions of daily food intake, based on the last 7 days of adaptation to deprivation.) The $S$ was removed immediately after eating the food pellet(s) or, if incorrect, after $5 \mathrm{sec}$. There was an approximately 3 -min ITI. Training was continued to a criterion of at least 8 correct spatial responses out of 10 over 2 successive days. After reaching this criterion, Ss were given daily 10-trial reversals for 15 days. The number of errors per reversal was recorded.

\section{RESULTS}

Gerbils and rats did not differ in learning the spatial discrimination. (Mean days to criterion were 2.8 and 2.1 for gerbils and rats, respectively; $t=0.36$.) There was a species difference, however, in performance on successive spatial reversals $(\mathrm{F}=11.10, \mathrm{df}=1 / 8, \mathrm{p}<.05)$. Reversal data were analyzed using a species by sex by reversals repeated-measures design analysis of variance. Reversals was the only other (than species) reliable source of variation $(\mathrm{F}=4.94, \mathrm{df}=1 / 14, \mathrm{p}<.01)$. In fact, Fs $<1.00$ were obtained for all other sources of variation. The percent errors per reversal are summarized in Fig. 1 for each species over successive reversals. As can be seen from Fig. 1, there was a slight improvement in performance over the 15 reversals for both species. but gerbils made slightly more errors than rats. At asymptote, for example, gerbils were still making about three (30\%) errors per reversal, while rats were making only two (20\%) errors on the average.

\section{DISCUSSION}

Though Wise \& Parker (1968) found gerbils to be inferior to

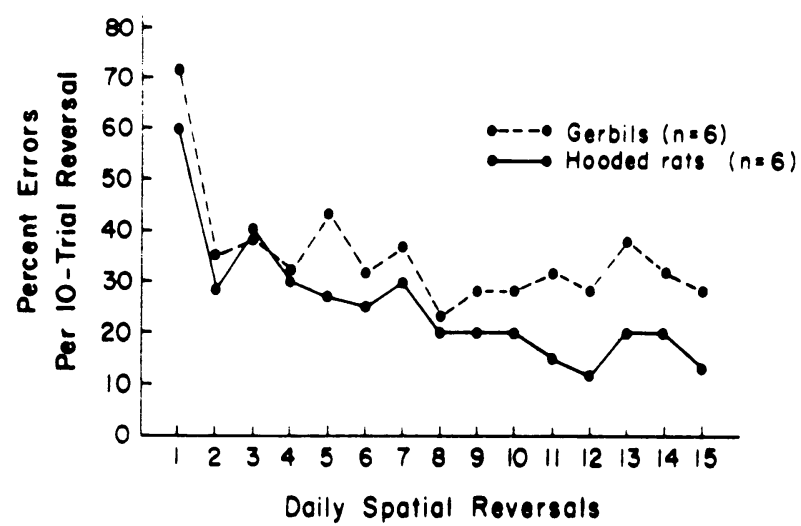

Fig. 1. The mean percent of errors on 15 successive reversals of a spatial discrimination in a T-maze by hooded rats and gerbils. 
albino rats in learning a brightness discrimination in a $Y$-maze. gerbils in the present study were not similarly inferior to hooded rats in learning a spatial discrimination in a $T$. They were inferior. however. in learning successive spatial reversals. Taken together. the Wise \& Parker (1968) and present findings suggest that gerbils perform well on appetitive maze learning tasks but may perform less well than rats (Rattus domesticus). Gerbils also were found to perform less well than albino rats on a one-trial passive avoidance task (Lippman et al. 1970). They noted that gerbil inferiority might have been due to competitive behavior. such as their greater tendency to explore (see also Glickman \& Hartz. 1964). In the present study, for example, gerbils of ten were observed to stop, sniff. and/or peer about, even after entry into the correct alley. The hoods. on the other hand, dashed directly to the foodcup.

Given their greater tendency to explore, gerbils might be expected to be as good or better than rats on tasks in which exploratory behavior would be advantageous (e.g., latent learning or signaled aroidance conditioning). Gerbils have been found to be as good or better than rats on active avoidance tasks. both signaled (Ashe \& McCain. 1972) and unsignaled (Boice et al. 1968: Powell. 1972). Thus, how well gerbils learn. relative to domestic rats. may depend on the extent to which the gerbil's greater tendency to explore and/or their greater activity level affects acquisition of a given task.

\section{REFERENCES}

Ashe, V. M., \& McCain, G. Comparison of one-way and shuttle avoidance performance of gerbils and rats. Journal of Comparative \& Physiological Psychology, 1972, 80, 293-296.

Boice, R., Boice, C.. \& Dunham, A. Role of docility in avoidance: Gerbils and Kangaroo rats in a shuttle box. Psychonomic Science, 1968, 10, 381-388.

Carey, M. A., \& Fischer, G. J. Spatial reversal learning in rats and gerbils. Paper presented (by title only) at the meeting of the Psychonomic Society, St. Louis, November, 1971.

Glickman, S., \& Hartz, K. Exploratory behavior in several species of rodents. Journal of Comparative \& Physiological Psychology, 1964, 58,101-104.

Lippman, L. G., Galosy, R. A., \& Thompson, R. W. Passive-avoidance learning in gerbils and rats. Journal of Comparative \& Physiological Psychology, 1970,73, 269-273.

Powell, R. W. Analysis of warm-up effects during avoidance in wild and domesticated rodents. Journal of Comparative \& Physiological Psychology, 1972, 78, 311-316.

Thompson, R. W., \& Lippman, L. G. Exploration and activity in the gerbil and rat. Journal of Comparative \& Physiological Psychology, 1972, 80, 439-448.

Wise, L. M., \& Parker, E. Discriminative maze learning in the Mongolian gerbil. Psychological Record, 1968, 18, 201-203.

(Received for publication February 28. 1973.) 